\title{
A self-consistent theory of nonequilibrium excitation transport in energetically disordered systems
}

\author{
Robert P. Parson ${ }^{\text {a) }}$ and Raoul Kopelman \\ Department of Chemistry, University of Michigan, Ann Arbor, Michigan 48104
}

(Received 5 September 1984; accepted 1 October 1984)

\begin{abstract}
The migration of incoherent excitations in energetically disordered systems is studied theoretically using a self-consistent diagrammatic approximation. Spatial diffusion and energy relaxation observables are related to the solutions of a nonlinear integral equation. Extensive numerical illustrations are given for two-component and multicomponent systems. In the latter, spatial transport is found to be highly dispersive (nondiffusive) over an extremely wide range of timescales, in accordance with results from simulations and experiments. The dependence of spatial and spectral transport properties upon the spatial range and the energy dependence of the intermolecular hopping rates is examined. Several measures of energy relaxation, including detailed probability distributions in energy space, relaxation-time spectra, and the nonequilibrium entropy are calculated and compared. The intimate relationship between spatial transport and energy relaxation is discussed in detail.
\end{abstract}

\section{INTRODUCTION}

Over the past few years a detailed, quantitative, and comprehensive theoretical picture of incoherent excitation transport in spatially disordered systems (solutions, random lattices, or amorphous structures) has emerged. An important component of this picture is provided by what we refer to, rather broadly, as effective medium theories. In these theories, perturbative expansions are partially summed by introducing frequency-dependent parameters which are determined by imposing self-consistency conditions; in a sense, a disordered system described by simple dynamical equations is replaced by an ordered one having more complicated dynamics. ${ }^{1-5}$ The effective medium results agree very well with exact calculations except in the immediate vicinity of a percolation edge, and even then provide a qualitatively reasonable, though quantitatively unsatisfactory, interpolation. They are also consistent with experimental results on a wide variety of systems ${ }^{6.7}$; in fact, such deviations as are observed are probably due to the inadequacy of the microscopic model rather than of the transport theory. ${ }^{7}$ Clearly, generalizations of this approach to more complicated systems would be of value. In this paper we develop an effective medium theory for systems in which both the energies and the positions of the excitation-conducting sites are random. Such systems are said to possess energetic, as well as topological, disorder. They have received much experimental attention in recent years. ${ }^{8-11}$

The microscopic description of the transport process in these systems can be formulated in the same way as for systems having only spatial disorder. One constructs a microscopic master equation for the site excitation probabilities, and considers the transition rates in this

\footnotetext{
a) Present address: Department of Chemistry, University of Washington,
} Seattle, Washington 98195 equation to be random variables whose distribution reflects the disorder. The major new effect that appears when energetic disorder is present arises from the asymmetry of the master equation: detailed balance implies that the forward and reverse rates connecting a pair of sites are related by a Boltzmann factor. At long times the excitation settles into the low-energy sites, so that spatial transport leads to energy relaxation. However, this relaxation also affects the rate of spatial transport since the distribution of site energies is not uniform; as relaxation takes place the higher-energy sites become increasingly difficult to reach so that the number of accessible sites decreases with time. This coupled relaxation process is of great importance for systems such as glasses, ${ }^{8.9}$ polymer aggregates, ${ }^{10}$ and orientationally disordered crystals, ${ }^{11}$ in which even at fairly high temperatures (certainly high enough to guarantee incoherent motion) the spread of site energies greatly exceeds the available thermal energy.

Our starting point is a natural generalization of the self-consistent diagrammatic expansion developed by Gochanour, Andersen, and Fayer, ${ }^{1}$ henceforth referred to as GAF. This theory is formulated in a continuum approximation, which should be valid when the average distance between sites is much larger than the minimum distance. In practice, the continuum approximation works well even when this restriction is violated, provided that an ad hoc short distance cutoff is imposed. ${ }^{7.12}$ (The most important effects of the cutoff, which merely removes the contribution to the overall transport of very rapid transfer between sites separated by very short distances, may be taken care of by using an effective transfer rate in place of the actual microscopic one.) We assume at the outset that spatial and energetic disorder are uncorrelated; this assumption is probably justified if the energy-conducting species are dispersed in an amorphous matrix, but is clearly false if they themselves form the amorphous structure. Since the transfer rates have an infinite range, 
there is a certain amount of self-averaging in the system which weakens the effect of such short-range correlations, so that ignoring them may be acceptable as a first approximation; nevertheless, this is perhaps the weakest assumption of the model. Once it is made, the diagrammatic approximation scheme of GAF can be applied, treating the site energy as an additional spatial coordinate. Since there is no translation invariance in this "energy coordinate," the resulting self-consistent equations are much more complicated than those which arise in the isoenergetic problem. They are nonlinear integral, rather than algebraic, equations. Nevertheless, they can be solved numerically without special difficulties, yielding the renewal function $G^{s}(E, t)$ (the probability that an excitation will be found on the site at which it was created, which obviously depends on the energy $E$ of the site) and a generalized, time-dependent transition rate $\Sigma\left(\mathbf{r} E, \mathbf{r}^{\prime} E^{\prime}\right)$ connecting points $\mathrm{r} E$ and $\mathbf{r}^{\prime} E^{\prime}$. From these one can calculate the spatially averaged excitation density at a specific energy, a quantity directly related to spectral diffusion experiments where only migration in energy is studied. One can also average over energies and obtain the effect of energetic disorder upon spatial transport by calculating a generalized diffusion coefficient. In Sec. IV we discuss results for both of these quantities. In order that this paper be reasonably self-contained, we repeat some material which has been previously presented in abbreviated form ${ }^{13}$; however, most of the present results (in particular, all of the numerical examples) are new.

While this work was being completed, a paper by Grünewald and co-workers appeared ${ }^{14}$ which presents a theory similar in many ways to ours. In their work, the effective medium concept is implemented through a selfconsistent decoupling of the renormalized perturbation expansion ${ }^{15}$ for the lattice Green function, the continuum limit being taken at a later stage. The theory invokes the same physical approximations as ours, but a detailed comparison is not easy; we give a general discussion in Sec. V. We believe that our theory and our calculations provide a useful complement to the work of Grünewald et al., especially since the GAF formalism upon which we build is probably more familiar to workers in the field of molecular excitation transport than the techniques used in Ref. 14.

\section{SELF-CONSISTENT DIAGRAMMATIC EXPANSION FOR ENERGETICALLY DISORDERED SYSTEMS}

The microscopic master equation for the site excitation probabilities $p_{n}(t)$ is

$$
\begin{aligned}
& p_{n}(t)=\sum_{n^{\prime}} W_{n n^{\prime}} p_{n}(t), \\
& W_{n n^{\prime}}=w_{n n}\left(1-\delta_{n n^{\prime}}\right)-\delta_{n n^{\prime}} \sum_{n^{\prime}} w_{n^{* n}} .
\end{aligned}
$$

The rate of transitions from site $n^{\prime}$ to site $n, w_{n n^{\prime}}$, depends upon the distance and energy mismatch between the sites:

$w_{n n^{\prime}}=v\left(\mathbf{r}_{n}-\mathbf{r}_{n^{\prime}}\right) \exp \left[-\frac{\beta}{2}\left(E_{n}-E_{n^{\prime}}\right)\right] \phi\left(\mid E_{n}-E_{n^{\prime}}\right)$.

The energy dependence consists of a detailed balance factor and an unspecified, in general, temperature-dependent symmetric factor $\phi$, which depends upon the details of the system-bath coupling. Since we assume that a continuum treatment is adequate, we will be interested in quantities defined at a point in space rather than on a specific molecule:

$p(\mathbf{r} E)=\sum_{n} \delta\left(\mathbf{r}_{n}-\mathbf{r}\right) \tilde{\delta}\left(E_{n}-E\right) p_{n}, \quad \tilde{\delta}(E) \equiv \frac{\delta(E)}{f(E)}$.

Here $f(E)$ is a normalized distribution of site energies. In the usual way, $p(r E)$ is understood to refer to a small volume in $r E$ space which is large enough to contain several molecules.

Observable properties can be related to the configuration averaged probabilities. In the continuum limit the averaging can be carried out as

$$
\langle p\rangle=V^{-N} \prod_{n=1}^{N} \int d \mathbf{r}_{n} \int d E_{n} f\left(E_{n}\right) p\left(\left\{\mathbf{r}_{n}, E_{n}\right\}\right),
$$

where $N$ is the number of molecules and $V$ the volume of the system; we will take the thermodynamic limit $N \rightarrow \infty, V \rightarrow \infty, N / V=\rho$. As in earlier work, ${ }^{1,2.12}$ we obtain the averaged probabilities from a Green function which is separated into diagonal (same-site) and nondiagonal (different-site) contributions:

$$
\begin{aligned}
G\left(\mathbf{r}, \mathbf{r}^{\prime}, E, E^{\prime}, u\right)= & \sum_{n n^{\prime}}\left\langle\delta\left(\mathbf{r}-\mathbf{r}_{n}\right) \delta\left(\mathbf{r}^{\prime}-\mathbf{r}_{n^{\prime}}\right) \tilde{\delta}\left(E_{n}-E\right) \tilde{\delta}\left(E^{\prime}-E_{n^{\prime}}\right)[u-\mathbf{W}]_{n n^{\prime}}^{-1}\right\rangle \\
= & \delta\left(\mathbf{r}-\mathbf{r}^{\prime}\right) \tilde{\delta}\left(E-E^{\prime}\right) \sum_{n}\left\langle\delta\left(\mathbf{r}-\mathbf{r}_{n}\right) \tilde{\delta}\left(E-E_{n}\right)[u-\mathbf{W}]_{n n}^{-1}\right\rangle \\
& +\sum_{n \neq n^{\prime}}^{\prime}\left\langle\delta\left(\mathbf{r}-\mathbf{r}_{n}\right) \delta\left(\mathbf{r}^{\prime}-\mathbf{r}_{n^{\prime}}\right) \tilde{\delta}\left(E-E_{n}\right) \tilde{\delta}\left(E^{\prime}-E_{n^{\prime}}\right)[u-\mathbf{W}]_{n n^{\prime}}^{-1}\right\rangle \\
\equiv & \delta\left(\mathbf{r}-\mathbf{r}^{\prime}\right) \tilde{\delta}\left(E-E^{\prime}\right) G^{s}(E, u)+G^{m}\left(\mathbf{r}-\mathbf{r}^{\prime}, E, E^{\prime}, u\right) .
\end{aligned}
$$

Note that $G^{m}$ is (because of averaging) translation invariant in $\mathbf{r}$ space but not in $E$ space, and that for the same reason the diagonal piece $G^{s}$ depends upon $E$ but not upon $\mathbf{r}$. We can thus remove the $\mathbf{r}$ dependence $b_{y} a$ Fourier transform: 


$$
\begin{aligned}
G\left(k, E, E^{\prime}, u\right) & =\int d r e^{-\mathbf{k} \cdot\left(\mathbf{r}-r^{\prime}\right)} G\left(r-r^{\prime}, E, E^{\prime}, u\right) \\
& =\tilde{\delta}\left(E-E^{\prime}\right) G^{s}(E)+G^{m}\left(k, E, E^{\prime}, u\right),
\end{aligned}
$$

but there is no analogous way to reduce the energy dependence because the distribution of energies is not uniform.

A diagrammatic analysis of the series expansion (in powers of $1 / u$ ) of the Green function may be developed precisely as in the isoenergetic case studied by GAF. We summarize this procedure very briefly, as it has been lucidly discussed in the original treatment. The various products of $w_{n n}$ 's which appear in the expansion are written as diagrams connecting sites labeled $n$ and $n^{\prime}$. The diagrams for the diagonal $G_{n n}$ and nondiagonal $G_{n n^{\prime}}$ parts of the unaveraged Green function are treated separately. A standard topological reduction procedure leads to a formal expression for $G_{n n^{\prime}}$ in terms of $G_{n n}$ and a twopoint self-energy function $S_{n n^{\prime}}$, defined as the sum of all irreducible diagrams (diagrams which cannot be broken by cutting a single interaction line) connecting sites $n$ and $n^{\prime}$. In more physical terms, $S$ is a generalized transition rate between the sites; it is defined to include all transitions among the two sites. The formal expression takes the form (henceforth we shall suppress the $\mathbf{k}$ and $u$ dependence when it is not needed):

$$
G_{n n^{\prime}}=\sum_{J=1}^{\infty} \sum_{\left\{n_{i}\right\}} G_{n n} S_{n n_{1}} G_{n 1 n 1} S_{n 1 n_{2}} \cdots S_{n נ n^{\prime}} G_{n^{\prime} n^{\prime}}
$$

Since each $S$ is itself a sum of diagrams, it actually refers to many site labels; by definition these labels do not occur in any other $S$ that occurs in each of the products in Eq. (2.7). In other words, each product describes a self-avoiding walk among the labels $n_{i}$. All revisitation events contribute to diagonal pieces or loops $G_{n n}$; since such loops occur, in general, in the irreducible parts $S$, each $S$ is a function of the $G$, and thus implicitly of the Laplace variable $u$. The crucial feature of the formal expansion (2.7) is that successive factors of $S$ are uncorrelated since the site energies and locations are independent random variables; thus the factors can be averaged independently. Identifying $\left\langle G_{n n}\right\rangle$ with $G^{s}$ and $\left\langle G_{n n^{\prime}}\right\rangle$ with $G^{m}$ and Fourier transforming one has

$$
\begin{aligned}
G^{m}\left(\mathbf{k}, E, E^{\prime}\right)= & \sum_{J=1}^{\infty} \rho^{J+1} \prod_{j=1}^{J} \int d E_{j} f\left(E_{j}\right) \\
& \times\left[G^{s}(E) \Sigma\left(\mathbf{k}, E, E_{1}\right) G^{s}\left(E_{1}\right)\right. \\
& \left.\cdots \Sigma\left(\mathbf{k}, E_{J}, E^{\prime}\right) G^{s}\left(E^{\prime}\right)\right],
\end{aligned}
$$

in which the self-energy $\Sigma$ is defined by the average:

$$
\begin{aligned}
\Sigma(\mathbf{k}, E, & \left.E^{\prime}\right) \\
= & (N-1)\left\langle\exp \left[i \mathbf{k} \cdot\left(\mathbf{r}_{n}-\mathbf{r}_{n^{\prime}}\right)\right]\right. \\
& \left.\times S_{n n^{\prime}} \tilde{\delta}\left(E-E_{n}\right) \tilde{\delta}\left(E^{\prime}-E_{n^{\prime}}\right)\right\rangle .
\end{aligned}
$$

Formally, the average runs over the positions and energies of all sites, but the values of the initial and final energies are constrained by the $\delta$ functions.
It is at this point that the lack of translation invariance in energy requires an extension of the theory. The presence of $E$-dependent factors in Eq. (2.8) prevents the simple summation carried out by GAF; instead, we must generate the series with the integral equation

$$
\begin{aligned}
G\left(\mathbf{k}, E, E^{\prime}\right) \equiv & \tilde{\delta}\left(E-E^{\prime}\right) G^{s}(E) \\
& +G^{s}(E) \Gamma\left(\mathbf{k}, E, E^{\prime}\right) G^{s}\left(E^{\prime}\right) \\
\Gamma\left(k, E, E^{\prime}\right)= & \rho \Sigma\left(k, E, E^{\prime}\right)+\rho \int d E^{\prime \prime} f\left(E^{\prime \prime}\right) \\
& \times \Sigma\left(\mathbf{k}, E, E^{\prime \prime}\right) G^{s}\left(E^{\prime \prime}\right) \Gamma\left(\mathbf{k}, E^{\prime \prime}, E^{\prime}\right)
\end{aligned}
$$

In general, $\Sigma$ is a functional of $G^{s}(E)$ as well as an explicit function of $E$ and $E^{\prime}$. Following the ideas of GAF once more, we can obtain explicit, albeit approximate, expressions for $G$ by choosing a convenient and accurate approximate form for the functional $\Sigma\left[G^{s}\right]$, and then determining $G^{s}$ by insisting that the approximation conserve total probability:

$$
\int d E f(E) G\left(k=0, E, E^{\prime}, u\right)=1 / u
$$

or using Eq. (2.10a):

$$
\begin{aligned}
\frac{1}{u G^{s}\left(E^{\prime}, u\right)}= & 1+\int d E f(E) G^{s}(E, u) \\
& \times \Gamma\left(k=0, E, E^{\prime}, u\right) .
\end{aligned}
$$

If Eq. (2.10) is substituted into Eq. (2.12) one obtains an exact relation between $\Sigma(k=0)$ and $G^{s}$ :

$\frac{1}{G^{s}\left(E^{\prime}, u\right)}=u+\rho \int d E f(E) \Sigma\left(k=0, E, E^{\prime}, u\right)$

This nonlinear integral equation determines the appropriate function $G^{s}(E)$ to be used with a given approximate functional $\Sigma\left[G^{s}\right]$. It is not a unique condition since it only involves the $k=0$ values of $\Sigma$; however, we will only be concerned with the region near $k=0$ and we will choose $\Sigma$ to be analytic in $k$ there. For a system which can be described (exactly or approximately) by a few discrete energies, the numerical solution of Eq. (2.13) is straightforward, although the computational labor increases rapidly with the number of energies retained.

The simplest approximate $\Sigma$ functional, analogous to the "two-body" approximation of GAF, is obtained by summing all diagrams in the $\Sigma$ series having exactly two sites. Physically this corresponds to retaining migration paths in which an excitation hops repeatedly within a pair of sites, occasionally making excursions (via $G^{s}$ ) to other sites but always returning to that site from which it left, and eventually moving on and never returning to that pair. The summation is straightforward (see Appendix A) and leads to 


$$
\Sigma\left(k, E, E^{\prime}\right)=\int d r e^{-i k r} \times\left(\frac{\exp \left[-\frac{\beta}{2}\left(E-E^{\prime}\right)\right] \phi\left(E-E^{\prime}\right) v(r)}{1+\left\{\exp \left[-\frac{\beta}{2}\left(E-E^{\prime}\right)\right] G^{s}\left(E^{\prime}\right)+\exp \left[+\frac{\beta}{2}\left(E-E^{\prime}\right)\right] G^{s}(E)\right\} \phi\left(E-E^{\prime}\right) v(r)}\right),
$$

which agrees with GAF when $E=E^{\prime}$. Note that $\Sigma\left(E, E^{\prime}\right)$ and $\Sigma\left(E^{\prime}, E\right)$ are related by a Boltzmann factor since the denominator is symmetric in $E, E^{\prime}$. Also, in this approximation $\Sigma\left(E, E^{\prime}\right)$ depends upon the value of $G^{s}$ at the two points $E, E^{\prime}$ only. In higher approximations, integrals over additional $E^{\prime \prime}$ will appear, greatly increasing the complexity of the calculation. Since the three-body calculation of GAF yielded only small corrections to the two-body calculation, we will restrict ourselves to the latter approximation.

For low densities and short times, a reasonable approximation for $G^{s}(E, t)$ can be obtained by discarding entirely the nondiagonal part of the master equation (2.1). This approximation is obviously not self-consistent, but it provides an excellent starting point for the iterative numerical solution of Eq. (2.13). The procedure for averaging $e^{\prime \mathbf{W}}$ when the nondiagonal part of $\mathbf{W}$ is neglected is well known ${ }^{16}$ and yields (Appendix B)

$G^{s}(E, t) \simeq \exp \left[-\rho \int d E^{\prime} f\left(E^{\prime}\right) \int d \mathbf{r}\left(1-e^{-t w\left(\mathbf{r}, E^{\prime}, E\right)}\right)\right]$,

which we call the diagonal approximation to $G^{s}$. The energy-independent version of this approximation has seen considerable use in non-self-consistent theories of energy transport. ${ }^{17}$

\section{TRANSPORT PARAMETERS}

In this section we relate the approximation scheme presented above to phenomenological transport properties. We first consider spatially averaged quantities, which describe migration in the energy coordinate only, and then proceed to spatially resolved quantities.

An obvious phenomenological description of the time evolution of the spatially averaged excitation density is a set of rate equations for an ensemble of phenomenological energy levels, i.e., a master equation in energy space:

$$
\dot{p}(E)=\sum_{E^{\prime}} K\left(E, E^{\prime}\right) p\left(E^{\prime}, t\right),
$$

in which $p(E)$ is the probability that a particle occupies energy level $E$ and $K\left(E, E^{\prime}\right)$ is the rate of transitions from $E^{\prime}$ to $E$. Such an equation is only true in special circumstances; however, a generalized version can be used under much broader conditions. If we define $p(E)$ as

$$
p(E)=\left\langle\sum_{n} \tilde{\delta}\left(E-E_{n}\right) p_{n}\right\rangle
$$

[note that we have factored out the density-of-states $f(E)$; the actual relative number of excitations having energy $E$ is given by $f(E) p(E)$ ], we can write

$$
\begin{aligned}
p(E, t)= & \sum_{E^{\prime}} G\left(k=0, E, E^{\prime}, t\right) p\left(E^{\prime}, t=0\right) \\
& + \text { (inhomogeneous term). }
\end{aligned}
$$

The inhomogeneous term arises from the arbitrary initial condition and vanishes if at $t=0$ the excitation density is uniform in $\mathbf{r}$ space. The Laplace transform of Eq. (3.3) can then be rearranged into a generalized master equation $^{18,19}$ :

$u p(E, u)=\sum_{E^{\prime}} K\left(E, E^{\prime}, u\right) p\left(E^{\prime}, u\right)+p(E, t=0)$.

The generalized master equation can be well approximated by a master equation, by replacing $K(u)$ by $K(u=0)$ (Markovian approximation), if the time scale for migration between energy levels is much longer than the time scale for migration within a level.

We can immediately relate the kernel $K(u)$ to the solutions of the self-consistent equation (2.13). From Eqs. (3.3) and (3.4), expressed in an obvious matrix notation, we have

$$
\mathbf{K}(u)=u \mathbf{1}-[\mathbf{G}(k=0)]^{-1},
$$

and from Eq. (2.10)

$$
\mathbf{G} \cdot \mathbf{f}=\mathbf{G}^{s} \cdot\left(\mathbf{1}+\boldsymbol{\Gamma} \cdot \mathbf{f} \cdot \mathbf{G}^{s}\right)
$$

so

$$
\mathbf{K}=\boldsymbol{u} \mathbf{1}-\mathbf{f} \cdot\left[\mathbf{G}^{s}\right]^{-1}+\boldsymbol{\rho f} \cdot \mathbf{\Sigma}(k=0) \cdot \mathbf{f}
$$

or, for $E \neq E^{\prime}$ :

$$
K\left(E, E^{\prime}, u\right)=\rho f(E) \Sigma\left(k=0, E, E^{\prime}, u\right) f\left(E^{\prime}\right),
$$

the interpretation of which is clear: the spatially averaged transition rate between levels depends upon the "intrinsic" generalized transfer rate $\Sigma$, integrated over space, and density-of-states factors $f(E)$. By using the self-consistency equation (2.13) one can readily show that Eqs. (3.4) and (3.7) conserve total probability. Once $\Sigma$ has been obtained from Eq. (2.13), the level populations can be obtained by solving the linear system (3.4). This procedure is quite general, requiring no separation of time scales since the $u$ dependence of $K$ is retained, and thus may be used to study the conditions under which the Markovian approximation is valid. Some numerical examples are discussed in the next section.

The calculation of spatially resolved quantities is more complicated. We begin by considering the time 
development of the mean-squared displacement, which serves to define a generalized diffusion coefficient:

$$
D(u)=\frac{u^{2}}{2 d}\left\langle\sum_{n} r_{n}^{2} G_{n 0}(u)\right\rangle
$$

or

$$
D(u)=\frac{-1}{2 d} \lim _{k \rightarrow 0} \nabla_{k}^{2} G(\mathbf{k}, u),
$$

where $d$ is the dimension of space. Again we go over to a matrix notation in energy indices:

$$
\mathbf{G}=\left[\mathbf{f} \cdot\left(\mathbf{G}^{s}\right)^{-1}-\rho \mathbf{f} \cdot \mathbf{\Sigma} \cdot \mathbf{f}\right]^{-1}
$$

We assume that $\Sigma(\mathbf{k})$ is analytic near $k=0$ and expand in $k$ :

$$
\begin{aligned}
\mathbf{G}(k, u) \simeq & \mathbf{G}(0, u)+[\mathbf{G}(0, u) \\
& \times \rho \mathbf{f} \cdot \tilde{\mathbf{D}}(0, u) \cdot \mathbf{f})] k^{2}+\mathbf{O}\left(k^{4}\right),
\end{aligned}
$$

where

$$
\tilde{\mathbf{D}}(\mathbf{k}, u) \equiv-\nabla_{k}^{2} \Sigma(k, u) .
$$

The energy-resolved generalized diffusion coefficient is then seen to be

$$
\begin{aligned}
D\left(E, E^{\prime}, u\right)= & \frac{u^{2}}{2 d} \int d E_{1} \int d E_{2} \\
& \times G\left(0, E, E_{1}, u\right) f\left(E_{1}\right) \tilde{D}\left(0, E_{1}, E_{2}, u\right) \\
& \times f\left(E_{2}\right) G\left(0, E_{2}, E^{\prime}, u\right) .
\end{aligned}
$$

Of particular interest is the average of this quantity over final, but not initial, energies:

$$
\begin{aligned}
D(E, u) \equiv & \int d E^{\prime} f\left(E^{\prime}\right) D\left(E^{\prime}, E, u\right) \\
= & u \int d E_{1} \int d E_{2} f\left(E_{1}\right) \tilde{D}\left(0, E_{1}, E_{2}, u\right) \\
& \times f\left(E_{2}\right) G\left(0, E_{2}, E, u\right) .
\end{aligned}
$$

This quantity is appropriate for spatially resolved experiments (direct measurements via transient grating or other methods) where the initial energy is selected, but detection is not energy resolved. The calculation of $D(E, u)$ is seen to involve the solution of an initial value problem in energy space as well as the calculation of the $\tilde{\mathbf{D}}$ matrix, which is obtained immediately from the self-energy $\Sigma$.

\section{NUMERICAL EXAMPLES}

\section{A. Two-component system}

The simplest nontrivial example of an energetically disordered system has exactly two discrete site energies. This case is interesting primarily because it allows one to investigate the behavior of the equations with minimal computational effort; however, it is obviously applicable to systems containing two energy-conducting species such as binary solutions or mixed crystals (in the latter case one must account for corrections to the continuum approximation).

If the two components are denoted as $(+)$ and $(-)$, the self-consistent equation (2.13) becomes

$$
\frac{1}{G_{ \pm}^{s}}=u+\rho\left[f_{+} \Sigma_{+ \pm} G_{+}^{s}+f_{-} \Sigma_{- \pm} G_{-}^{s}\right] .
$$

(If the microscopic rates $w_{+-}$leading from lower to higher energy sites are set equal to zero, a zero temperature, or deep trap limit, this becomes identical to the self-consistent trapping equation of Loring, Andersen, and Fayer. ${ }^{2}$ ) This equation is easily solved numerically; in all of our work we used a modified Newton-Raphson algorithm ${ }^{20}$ which avoids the explicit calculation of derivatives and requires fewer function evaluations than the standard one. We found that for most parameter $(\beta, u, \phi)$ choices the diagonal approximation, Eq. (2.15), made an excellent initial guess; at low temperatures it frequently failed, but this was remedied by continuing the solution down from high temperatures to low. To ensure that a reasonable choice was made among the multiple solution branches which may occur, we chose various initial $(u, \beta)$ values, obtained a solution starting from the diagonal approximation, and followed the solution branch in the $(u, \beta)$ plane by using each result as an initial guess for a nearby point. Nowhere did we find alternative solutions having positive $G^{s}(u)$; although we have not searched the entire parameter space, we can say that any alternative solutions must lie very far from the physically reasonable results that we have obtained. Our experience in this regard is similar to that described by Loring and Fayer ${ }^{2}$ for the simple trapping model, and suggests that the two-body self-consistent approximation may have analytic properties similar to those that have been proved for the coherent medium approximation. ${ }^{21}$

We are particularly interested in the time scale on which $G^{s}(t)$ decays, as this determines the times for which the Markovian approximation is valid. The resulting twolevel rate equation dynamics is sufficiently familiar not to require explicit discussion here, although we emphasize that the value of $\Sigma_{+-}(u=0)$ gives the spatially averaged rate of energy transfer between components, and that when the Markovian approximation fails one can still obtain the level populations by solving a $2 \times 2$ linear system and inverting the Laplace transform. For our examples, we chose an isotropic transition multipolemediated transfer rate:

$$
v(r)=(1 / \tau)(R / r)^{s},
$$

where $\tau$ is the mean time required for a hop of a distance $R$. We consider here the most common case of dipoledipole (Förster) transfer, $s=6$, in three dimensions, and choose length and time scales such that $R=\tau=1$ and an energy scale in which $E_{+}-E_{-}=2$. We also set $\rho$ $=1$ (given the time dependence, the density dependence can be obtained from a scaling argument ${ }^{12}$ ). The twobody self-energy becomes 
$\Sigma\left(k=0, E, E^{\prime}, u\right)=\frac{\sqrt{\phi\left(E-E^{\prime}\right)} e^{-\beta}}{\left[e^{-\beta} G^{s}\left(E^{\prime}\right)+e^{+\beta} G^{s}(E)\right]^{1 / 2}}$.

In an experimental system, one would obtain $\phi(E)$ from the spectra of individual molecules through the Förster prescription. ${ }^{22}$ For these examples, we have set $\phi(0)=1$ and chose $\phi(2)$ equal to 1.0 and 0.1 ; the latter choice reflects the expectation that the resonant process will be faster. We consider the case of equal component concentrations, $f_{+}=f_{-}=0.5$.

In Figs. 1 and 2 we show the function $G_{ \pm}^{s}(t)$, the probability of an excitation being found at the origin, as determined by numerical Laplace inversion ${ }^{23}$ of the solution to Eq. (4.1). As one of course expects, this probability is greater if the initial site is in the lower level, and the asymmetry increases with decreasing temperature. Decreasing the rate of nonresonant transfer (Fig. 2) slows down all processes, but primarily affects the upper level; in the lower level, nonresonant transfer is already opposed by the Boltzmann factor so that decreasing $\phi(2)$ has only minor effects, particularly at the lower temperature. Our basic time unit corresponds roughly to the time required for a transfer to a site separated by an average intermolecular distance; one can see that $G_{ \pm}^{s}(t)$ has decayed essentially to zero by this time, so that most of the transport does occur in the Markovian regime and a simple rate equation description is appropriate for this example.
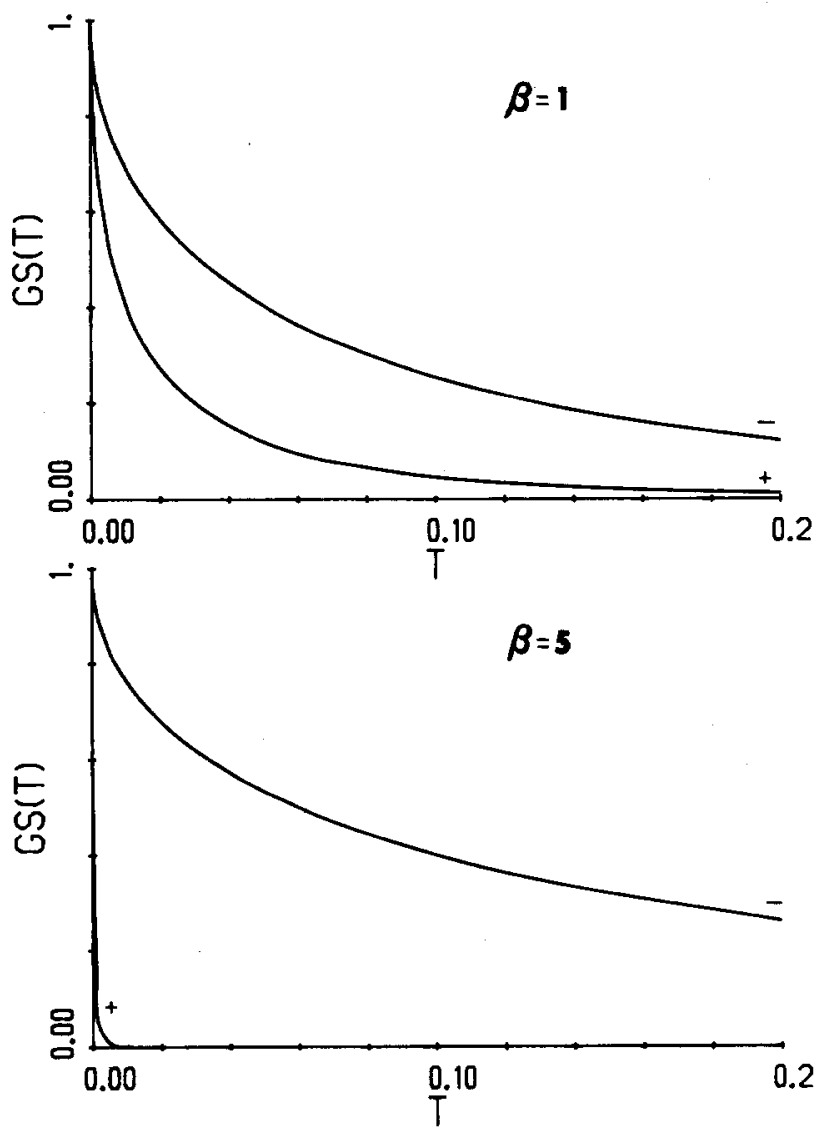

FIG. 1. Renewal functions for two-component systems. The functions $G_{-}^{s}(t)$ and $G_{+}^{s}(t)$ are plotted against time for $\beta=1$ (upper graph) and $\beta$ $=5$ (lower graph), $f_{+}=f_{-}=0.5$, and a mismatch parameter $\phi=1.0$.

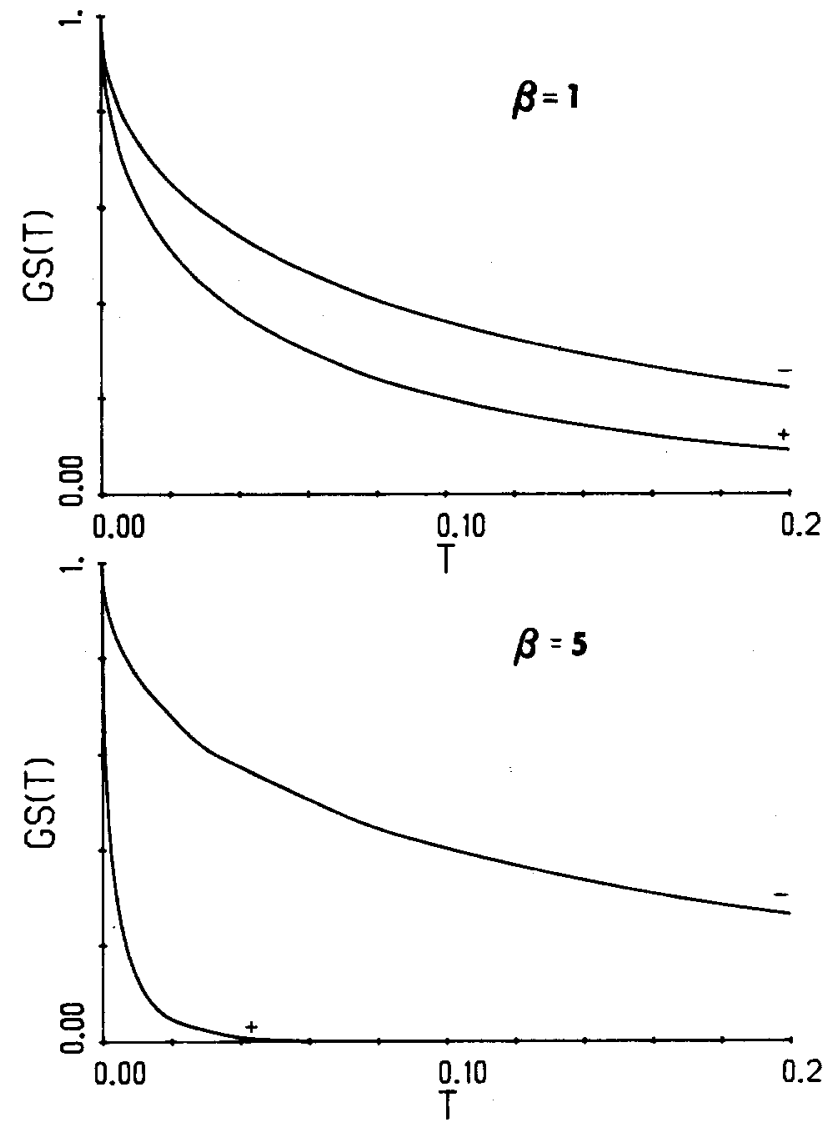

FIG. 2. Same as Fig. 1, except that $\phi=0.1$.

\section{B. Multicomponent system}

The major application of this theory is to systems having a continuous distribution of energies. In this case, the nonlinear integral equation (2.13) must be solved by discretizing the energy coordinate and solving the resulting nonlinear system. Since the computational labor increases rapidly with the number of discrete levels retained, it is worthwhile to minimize this number by using a Gaussian quadrature rule appropriate for the distribution $f(E)$. For our examples we used a Gaussian $f(E)$ with a 20-point Gauss-Hermite rule. ${ }^{24}$ In experimental applications, one would obtain $f(E)$ from the system's absorption spectrum.

The calculation of level populations involves two steps: first Eq. (2.13) is solved for $G^{s}(E, u)$, from which $K\left(E, E^{\prime}, u\right)$ is obtained via Eq. (3.8), and then the generalized master equation (3.4) is solved for $p(E, u)$ for a given initial condition. The methods used for the first step are the same as those used for the two-component case. The second step requires some care, particularly for small $u$ where the matrix $[u-\mathbf{K}(u)]$ becomes severely ill conditioned. This problem arises from the zero eigenvalue, associated with conservation of total probability, of the $\mathbf{K}$ matrix, and is easily fixed by a simple transformation. The transformed system still has some very small eigenvalues (for reasons which we shall describe), and to ensure stability of our results we used iterative refinement ${ }^{25}$ when solving the system. This combination gave satisfactory results for all of our examples. The complete calculation of $p(E, t)$ for ten values of $t$ required about $15 \mathrm{CPU}$ 
seconds on the Amdahl 5860 at the University of Michigan.

The 20-point quadrature is entirely adequate for relatively poorly resolved initial conditions, such as one obtains in experiments using filtered incoherent excitation. In order to interpret high-resolution experiments, such as one would carry out with laser excitation, one needs a high-resolution calculation. This is prohibitively expensive to do directly (the computation cost varies as the cube of the number of discrete levels); however, one can avoid this by noticing that the computationally intensive part of the calculation, which is the solution of Eq. (2.13), results in a single function $G^{s}(E)$. This function varies smoothly with $E$, so one may compute it using the 20point quadrature and then interpolate intermediate values. (We interpolated in the $\log$ of $G^{s}$ as this varies more slowly than $G^{s}$ itself.) The interpolated function is then used to construct an enlarged $\mathbf{K}$ matrix, from which the populations of the enlarged system are calculated. By this procedure, we were able to increase the number of discrete levels in the final calculation to 100 , while increasing the computation cost by only a factor of 2 .

An alternative way to characterize the energy-space dynamics is to calculate a spectrum of relaxation times. Since the energy-space density is given by $f(E) p(E)$, the appropriate eigenvalues are those of the matrix $\mathbf{K}(u) \cdot \mathbf{f}^{-1}$, rather than those of $\mathbf{K}(u)$. The spectrum yields a quantitative measure of such ideas as "separation of time scales," and also describes, through its $u$ dependence, the approach to Markovian behavior: when most of the eigenvalues become approximately independent of $u$, the transport can be described by Markovian rate equations. (To be more precise, one should examine the eigenvectors, and associate regions of $E$ space with particular eigenvalues, but this is rather cumbersome.) This does not imply that all the elements of the $\mathbf{K}(u)$ matrix are independent of $u$; many of these elements are irrelevant for the bulk of the transport (as an example, consider a system containing perfect traps. Since there is no transport among the traps, their "dynamics" is never Markovian; the diagonal Green function $G_{\text {trap }}^{s}(t)$ never decays to zero. However, the transport among the nontrapping sites may be Markovian. This will not be apparent in the energyspace generalized master equation since all transfer rates involving the traps will be $u$ dependent; by computing the eigenvalues one sorts out the Markovian and nonMarkovian portions of the transport.) The eigenvalue spectrum, even in the relatively coarse discrete approximation used here, is a very useful and easily calculated invariant measure of spectral migration in the system.

Finally, once one has a solution (in Laplace space) of the spectral transport problem for a given initial condition, one can calculate the spatial diffusion coefficient for this same initial condition from Eq. (3.15). We chose to leave these results in Laplace space since $D(E, u)$ sometimes varies very slowly with $u$ (this is of course the signature of classical diffusion), so that Laplace inversion is very ill conditioned. The important features are readily seen in the Laplace domain since any deviation of $D(u)$ from a constant value indicates nondiffusive behavior.
We have carried out calculations for three-dimensional systems having a Gaussian distribution of site energies, whose $1 / e$ half-width defines our basic energy and temperature scale, and transition-multipole mediated intersite excitation transfer rates; we have considered specifically the cases of dipole-dipole and octupole-octupole interactions $[s=6$ and 14 , respectively, in Eq. (4.2)]. The octupole contribution is important for molecules such as naphthalene or benzene which have weak or vanishing transition dipole and quadrupole moments. The remaining system parameter is the symmetric mismatch factor $\phi(E)$. It is difficult to determine what this should be a priori; in principle, it is given by the Förster spectral prescription, ${ }^{6.22}$ but this involves the absorption and emission spectra of individual molecules in the medium. It is quite impossible to separate the spectrum of an individual molecule, particularly the multiphonon wing which is responsible for off-resonant transfer, from the inhomogeneous broadening described by $f(E)$. In lieu of any detailed understanding, we have selected a form for $\phi(E)$ which agrees with the intuitive idea that resonant transfer should be rapid:
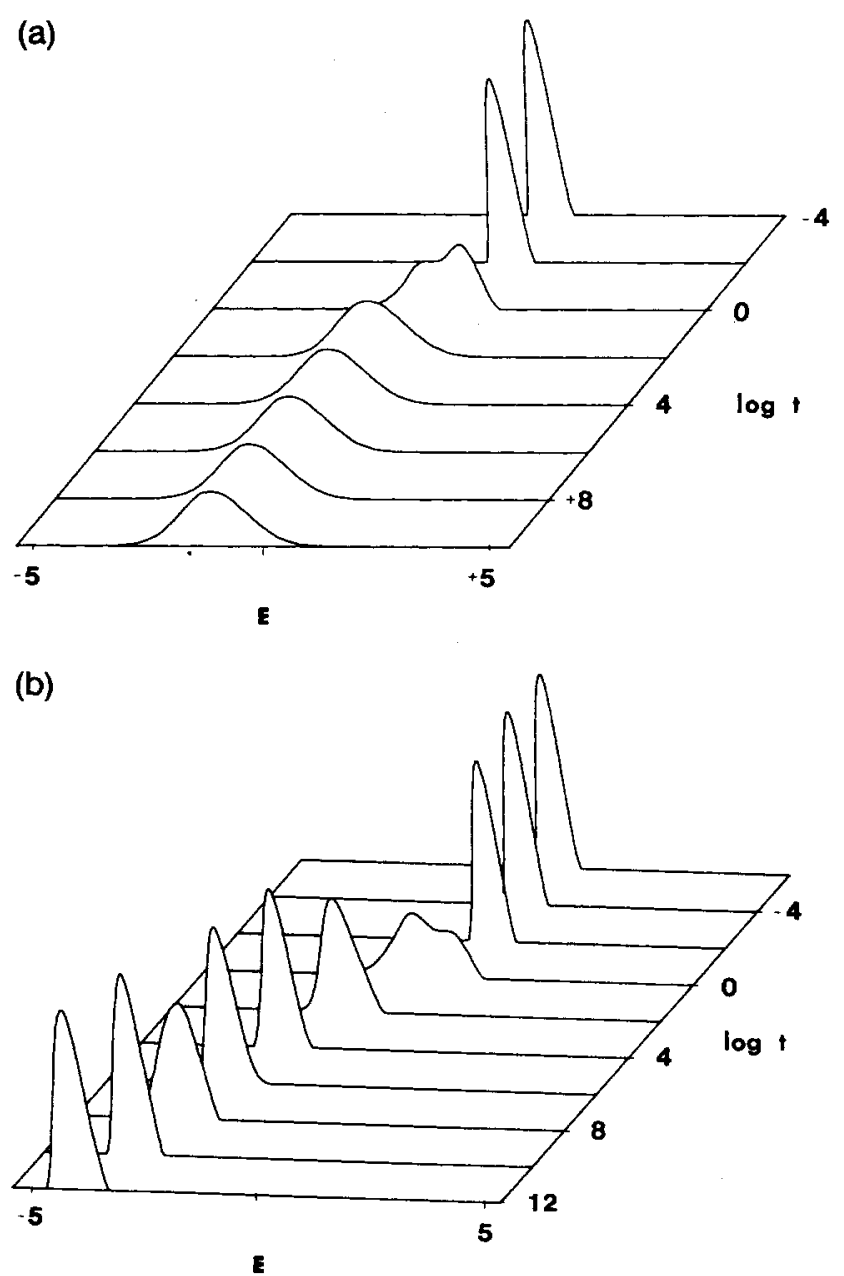

FIG. 3. Spectral migration in an energetically disordered system. The energy-space density $f(E) p(E, t)$ is shown for a system having dipolar transfer rates and a mismatch parameter $\phi=0.01$, and (a) $\beta=1$, (b) $\beta=9$. The curves are rational (not cubic) splines fitted through the 20 discrete energies; they have been adjusted to be height true (no extraneous inflections), but are not necessarily area true. 


$$
\phi\left(E-E^{\prime}\right) \exp \left(+\frac{\beta}{2}\left|E-E^{\prime}\right|\right)= \begin{cases}1, & \left|E-E^{\prime}\right|<\Delta \\ \phi, & \left|E-E^{\prime}\right|>\Delta,\end{cases}
$$

where $\phi$ is a number $\leq 1$. Outside of the "resonant width" $\Delta$, the intersite transfer rate (2.2) is independent of $E$ except for the Boltzmann factor. We find that the qualitative features of our results are not much affected by the value of $\Delta$ or by choosing different forms for $\phi(E)$ consistent with the same ideas. The results presented here use $\Delta=0.25$, which is the smallest energy step in the system, so that transfers within a discrete level are treated differently from transfers to other levels.

Our results for the energy-space density $f(E) p(E, t)$ are shown in Figs. 3-7. The most notable feature of these results is the establishment of a local (in energy space) equilibrium distribution, followed by a slow relaxation of this distribution to lower energies. (In Figs. 3 and 5, the detailed shape of the distribution is not very meaningful towards the ends of the energy scale because the discrete energy levels are sparse there. The location and overall width are significant.) Eventually, a static global equilibrium is established, but at low temperatures the time scale associated with this is enormous. The physical reason for this is clear: as discussed in the introduction the density of accessible sites decreases as the excitation
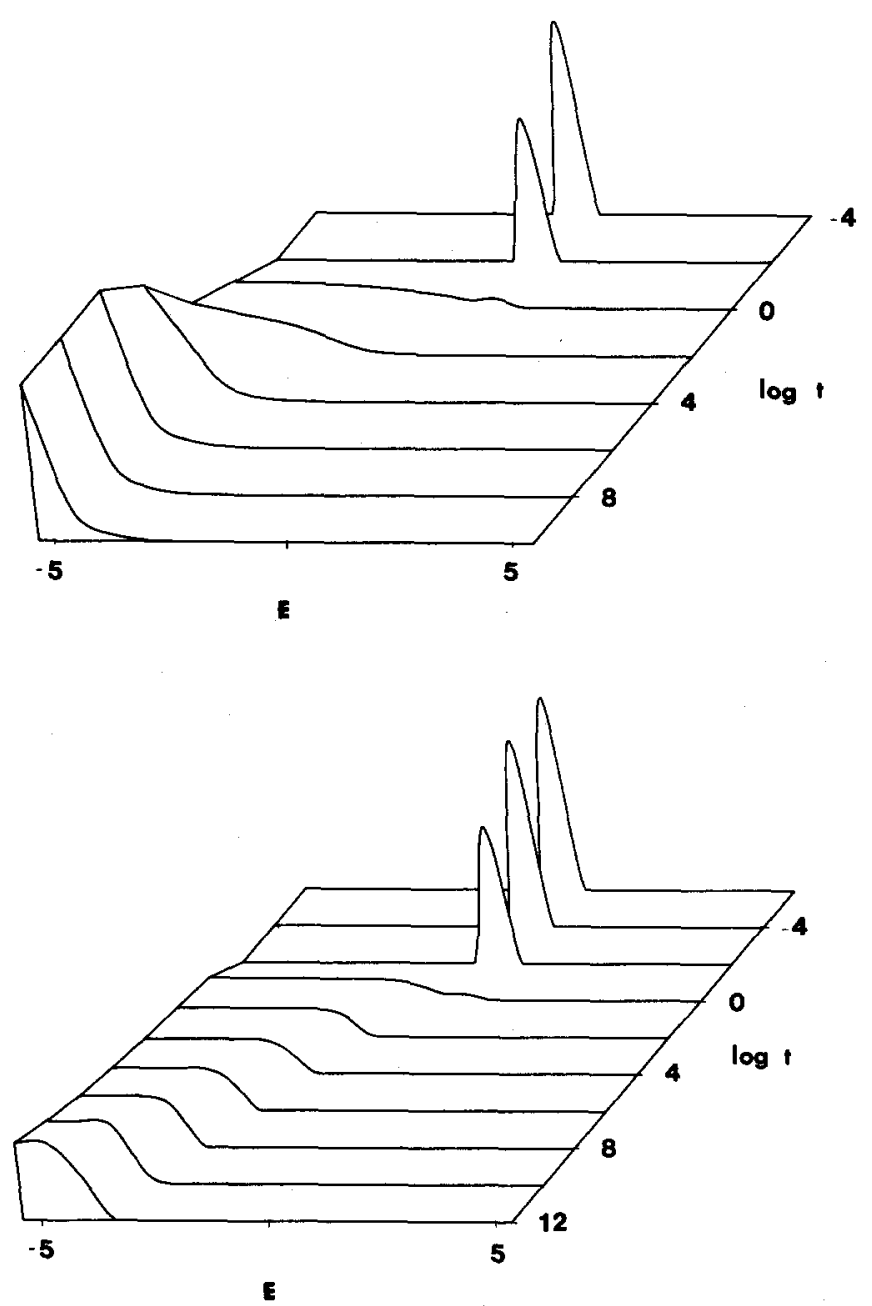

FIG. 4. The level population $p(E, t)$ (energy-space density divided by density of states) is shown. All parameters are as in Fig. 3.
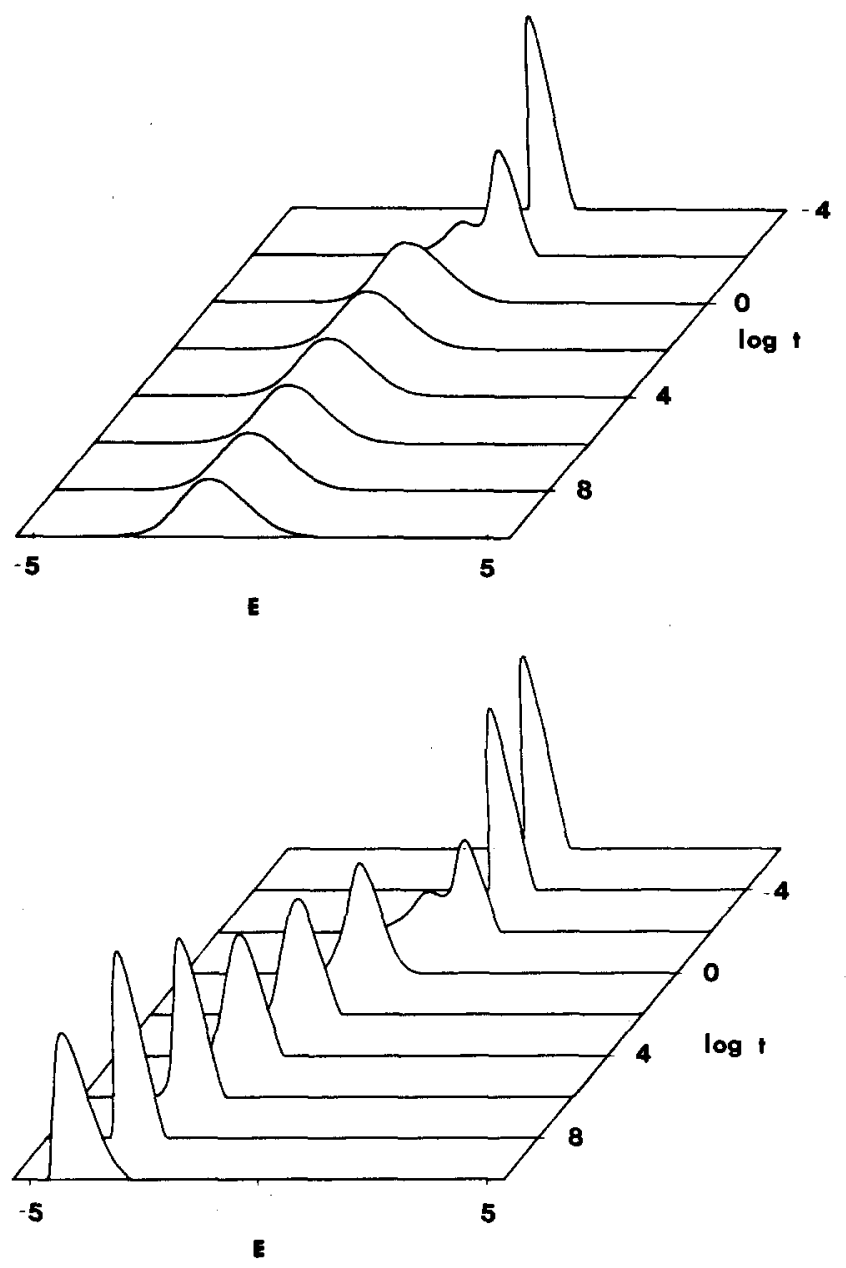

FIG. 5. Same as Fig. 3, except that $\phi=1.0$.

packet relaxes so that the relaxation process decelerates. At very low temperatures excitations get trapped for very long times in moderately low energy sites, and are unable to reach their equilibrium energies. This is made especially clear in Fig. 4, where we have factored out the densityof-states. We note that while molecular singlet excitations have short lifetimes and may thus never undergo signifcant energy relaxation in dilute systems, the lifetimes of triplet excitations are very long (on the order of $1 \mathrm{~s}$, which is to be compared to a transfer time measured in picoseconds), so that much of Figs. 3-5 may be accessible. We have not dealt with the exchange-mediated transfer which characterizes triplet excitations, but we expect the results to be very similar, since the dipole and octupole cases do not differ greatly.

Another important feature of our results is the insensitivity of the long-time behavior to initial conditions. This is again to be expected on physical grounds; indeed, it is implicit in the concept of local equilibrium we used above. The effect is easily seen in the high-resolution plots (Figs. 6 and 7); the initial narrow peak is essentially replaced by a broad distribution which swells up from the background. We emphasize that the local equilibrium will only be established if the excitation lifetime is long; steady state observations of short-lived excitations will certainly be sensitive to initial conditions. 

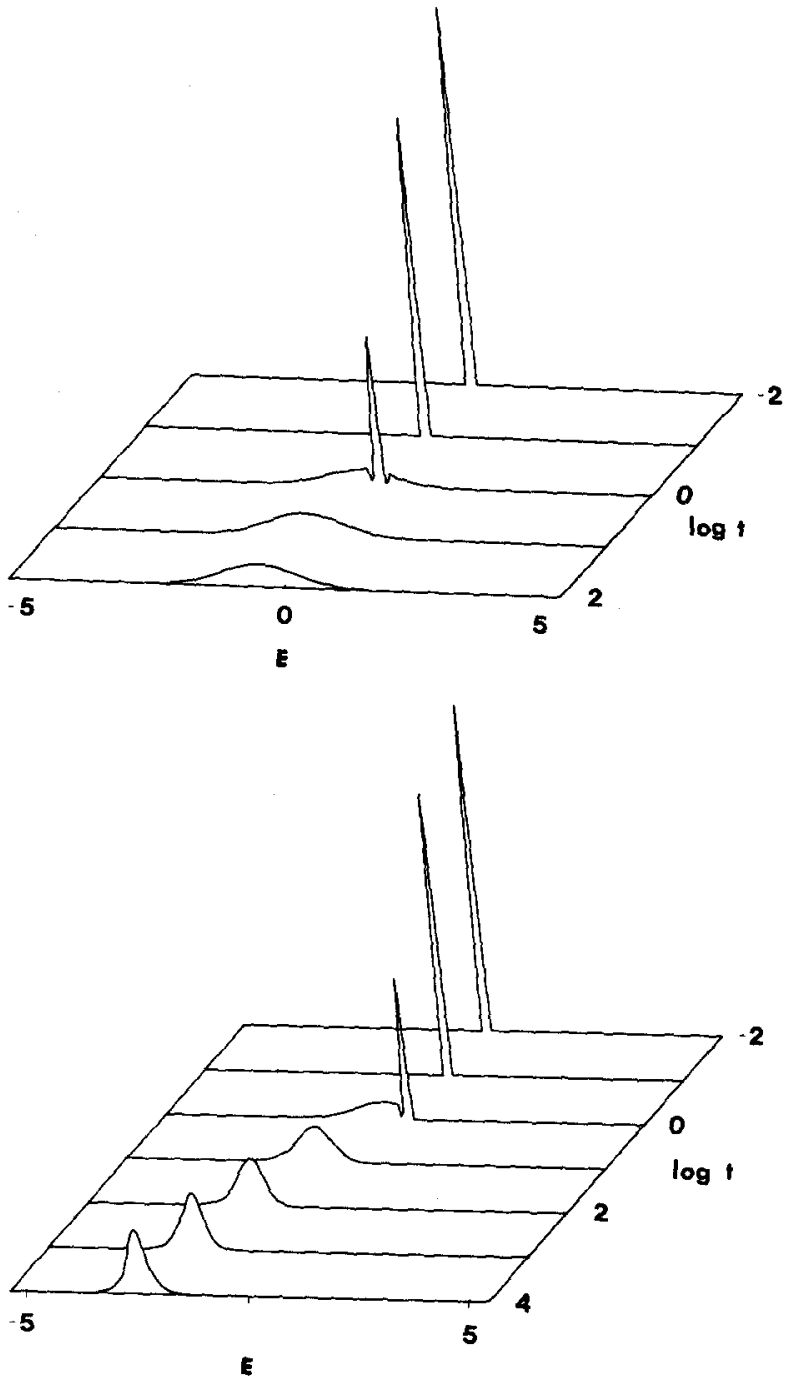

FIG. 6. High resolution spectral migration. Spectral migration of a narrow initial excitation packet, calculated using the interpolation procedure described in the text, is shown. For this example, 96 discrete energies were used. The parameters are those of Fig. 3.

Figure 8 shows sample eigenvalue spectra. Using the criterion discussed above, one can see that at high temperatures Markovian dynamics is established for $u<1$. One expects this: the short-time dynamics is dominated by direct transfer and by motion within small clusters of sites, but once enough time has elapsed for transfer over typical intersite distances, these correlated transport processes become less important. At very low temperatures, the lowest-lying eigenvalues take longer to settle down. This is also easily rationalized: the dynamics of an excitation in the low-energy tail of the distribution is very much like the dynamics in an extremely dilute system, since the density of accessible sites is so low. By a wellknown argument, ${ }^{12}$ one can relate long-time dynamics in a low-density system to short-time dynamics in a highdensity system. The enormous spread of time scales evident in Figs. 3-7 is made obvious here by the range of relaxation times. In contrast, a rectangular distribution of energies [Fig. 8(c)] yields a rather compact set of eigenvalues, since in this system there is no deceleration mechanism; excitations fall quickly into the lowest levels.
It is useful to have a one-parameter measure for the extent of energy relaxation as a function of time. One such measure is the average excitation energy, the first moment of $f(E) p(E)$. This contains, however, no information about the shape of the distribution, and is thus insensitive to such effects as the narrowing of the excitation packet which can be seen in Fig. 6. [One can ascribe this narrowing to the rapid decrease of the relaxation rates $K\left(E, E^{\prime}\right)$ as $E$ moves into the band tail; the excitations tend to collect together at a given $E^{\prime}$ before relaxing to a lower one. A subsequent broadening must eventually occur, but only when the final equilibrium average energy is reached.] A quantity which is sensitive to all moments of the distribution is the entropy functional or Liapunov functional $H(t)^{26}$ :

$$
H(t) \equiv \int d E f(E) p(E, t) \ln \left[\frac{f(E) p(E, t)}{P_{\mathrm{eq}}(E)}\right]
$$

where

$$
P_{\mathrm{eq}}(E) \equiv \frac{f(E) e^{-\beta E}}{\int d E f(E) e^{-\beta E}} .
$$
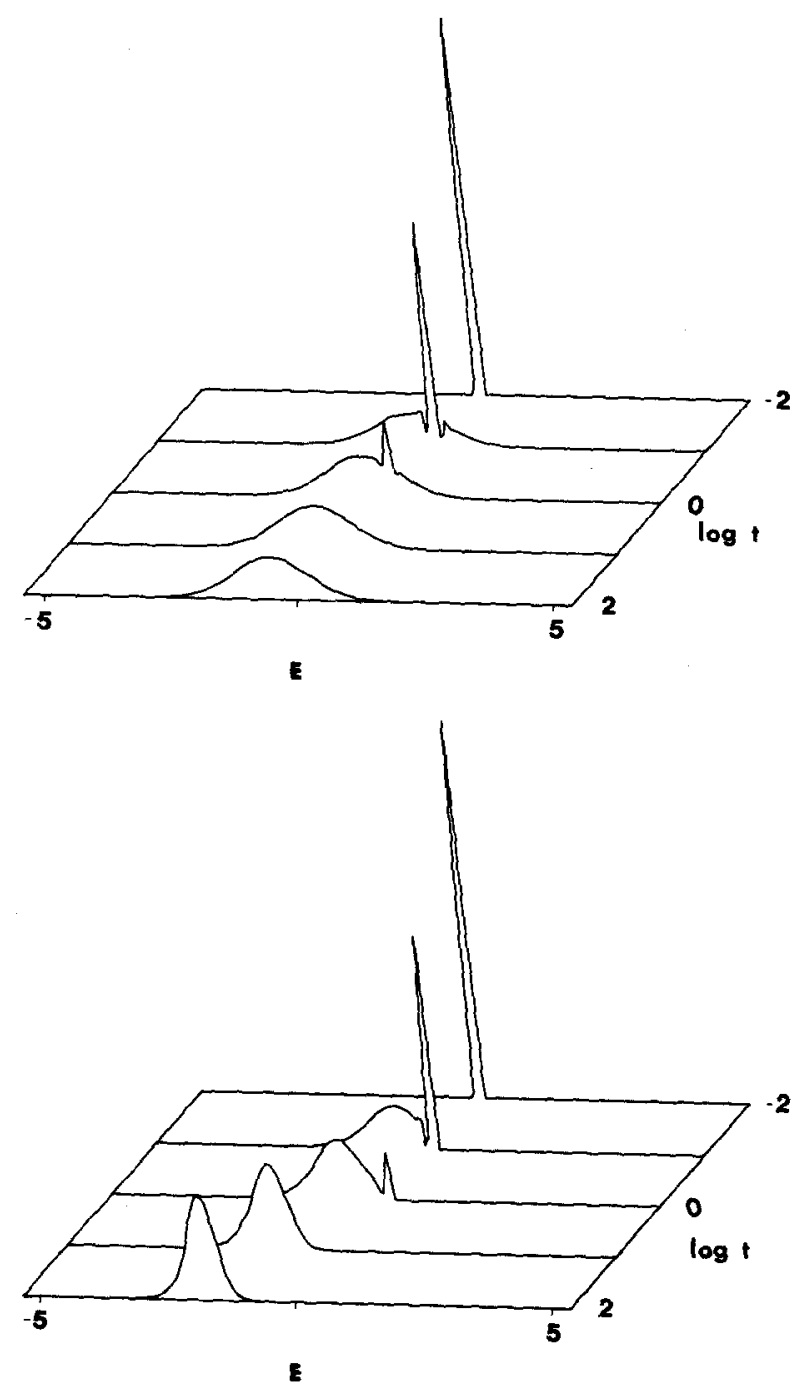

FIG. 7. Same as Fig. 6, except that octupole-octupole transfer rates are used. (The rates are scaled so that dipole and octupole interactions give the same rate at the Förster radius.) 
(a)
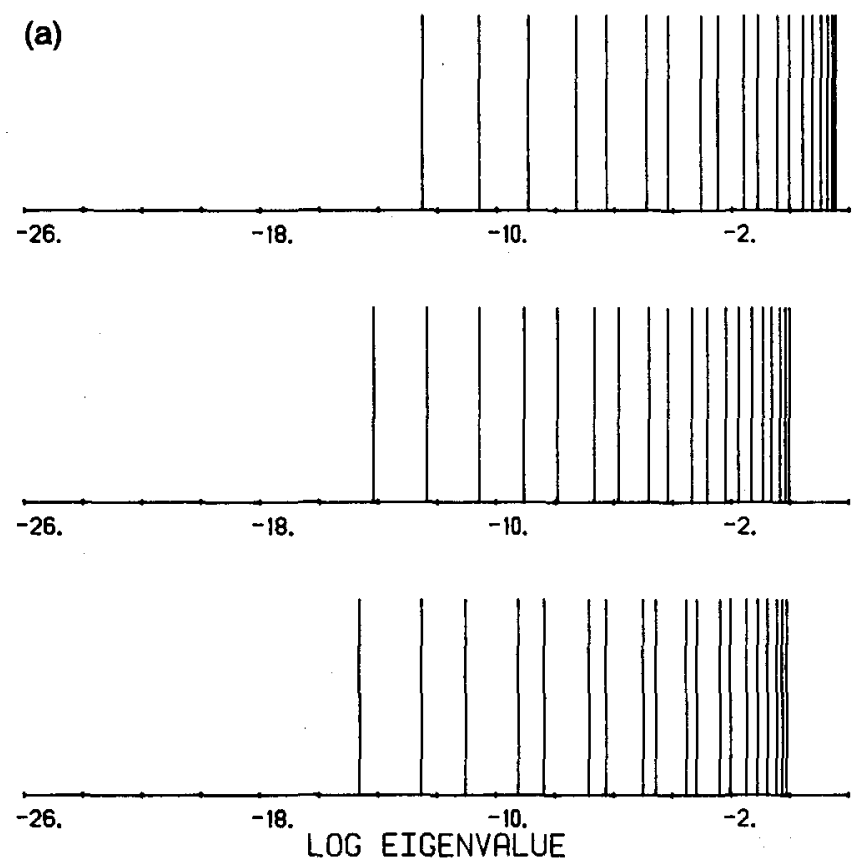

(c)

$-18 . \quad-10$.

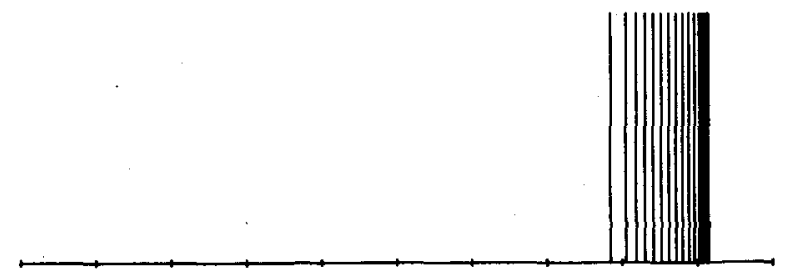

$-18 . \quad-10$.

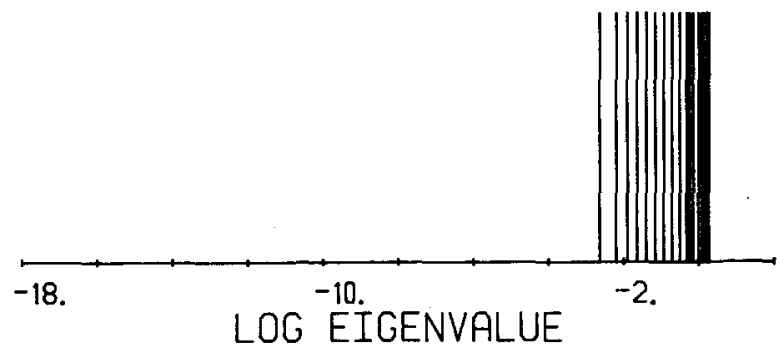

By construction, $H(t)$ decays monotonically to zero (this is strictly true for a Markovian master equation, and we do not expect the small non-Markovian effects in our system to alter this). In Fig. 9, we show that at low temperatures the decay of $H(t)$ is given approximately by a power law over the range $t=1$ to $t=10^{8}$. This is another illustration of the wide range of time scales characteristic of energetically disordered systems.
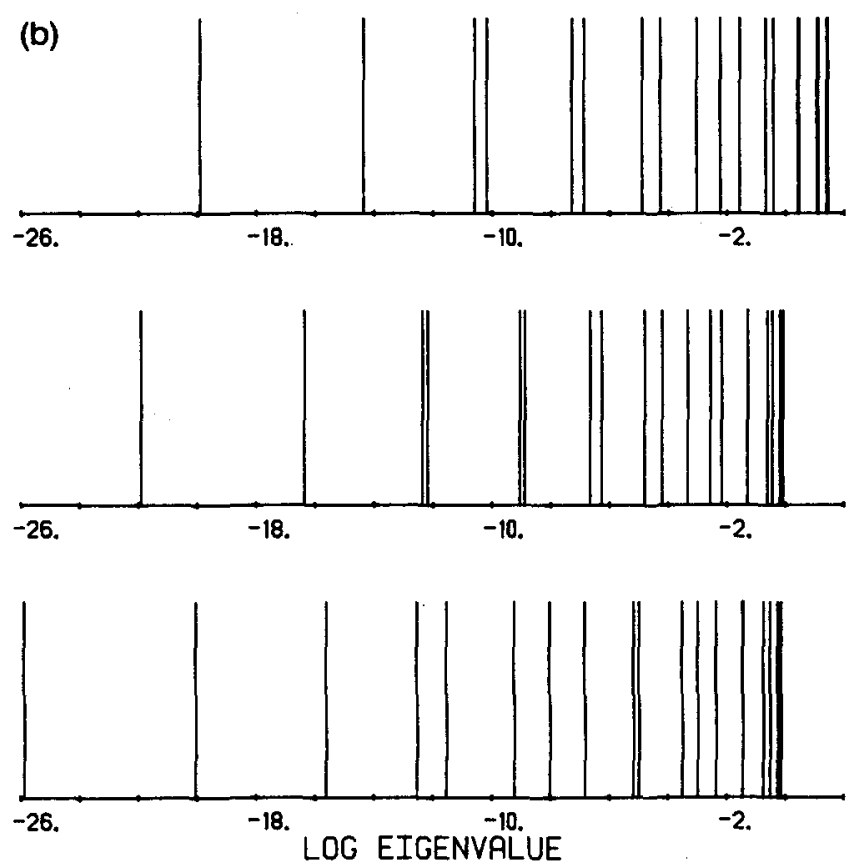

FIG. 8. Energy relaxation spectra. The nonzero eigenvalues of the matrix $\mathbf{K}(u) \cdot \mathbf{f}^{-1}$ (see the text) are shown. For this example, $\phi=0.01, \beta=1$ (a) and 9 (b), and (top to bottom) $u=10^{+4}, 1$, and $10^{-12}$. In (c), a rectangular density of states, having $\beta=5$ in units of its half-width, was used.

Our most interesting results involve spatial transport. In Figs. 10 and 11 we show the spatial diffusion coefficient, obtained from Eq. (3.15), as a function of the Laplace variable $u$ for several parameter choices. One sees immediately that at low temperatures the transport is not diffusive on any reasonable time scale, in marked contrast to the results obtained for systems having only spatial disorder. ${ }^{1-6}$ A given value of $D(u)$ corresponds roughly to 


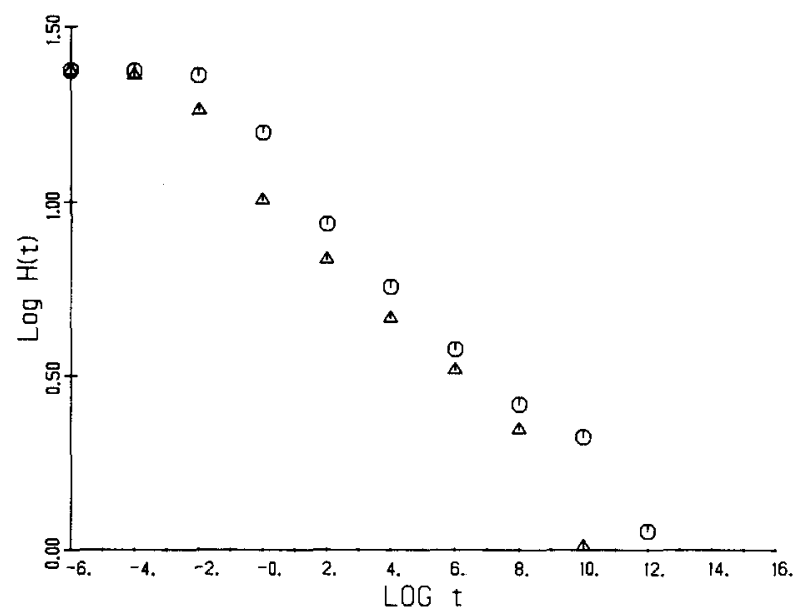

FIG. 9. The decay of the nonequilibrium entropy $H(t)$ calculated from the distributions displayed in Figs. 3(b) (circles, $\phi=0.01$ ) and 5(b) (triangles, $\phi=1.0$ ) is shown.

the diffusion constant associated with times $\sim 1 / u$; more precisely, $D(u) \sim u^{1-x}$ implies $\left\langle r^{2}(t)\right\rangle \sim t^{x}$. At short times, the limiting Haan-Zwanzig behavior ${ }^{12}$ :

$$
\left\langle r^{2}(t)\right\rangle \sim t^{(d+2) / s}
$$

is approached in all cases. However, at $t \sim 1$, there is a crossover to highly dispersive behavior; in many cases the dynamics is farther from classical diffusion at long times than at short. The figures demonstrate that at low temperatures the form of the long-time behavior is rather insensitive to several of the obvious system parameters. This insensitivity is readily rationalized in most cases: from Figs. 3-7 one may surmise that the spatial transport is locally diffusive, with a diffusion constant depending upon location of the excitations in energy space. Because of the deceleration of the energy relaxation, excitations created at $E=0$ catch up with those created at $E$ $=-2.25$, so that the curves in Fig. 10 corresponding to

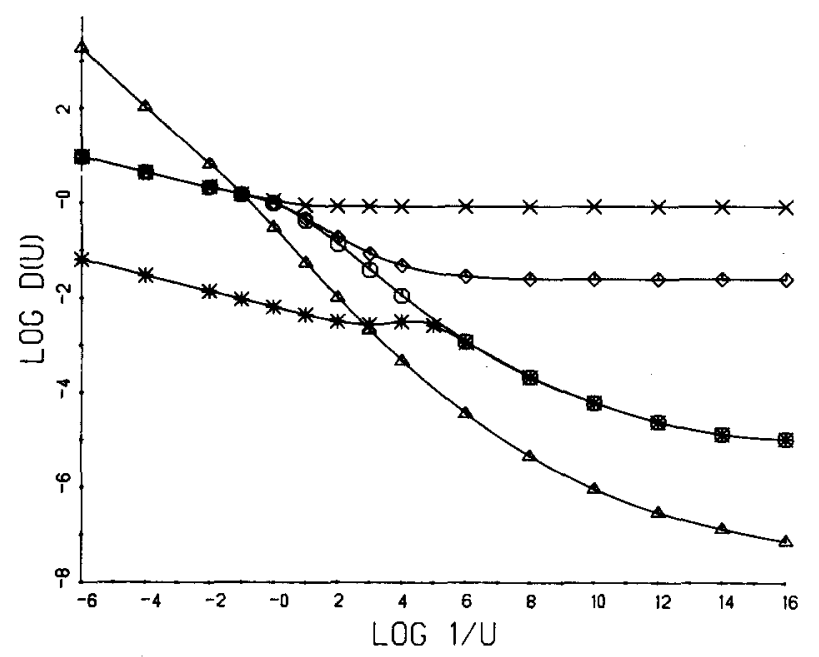

FIG. 10. Spatial migration in an energetically disordered system. The generalized diffusion coefficient $D(E, u)$ is shown as a function of the imaginary frequency $u$ for: $x: \beta=1, E=0$, dipolar transfer; diamonds: $\beta=5, E=0$, dipole; circles: $\beta=9, E=9$, dipole; triangles: $\beta=9$, $E=0$, octupole; stars: $\beta=9, E=-2.25$, dipole. $\phi=0.01$ in all cases.

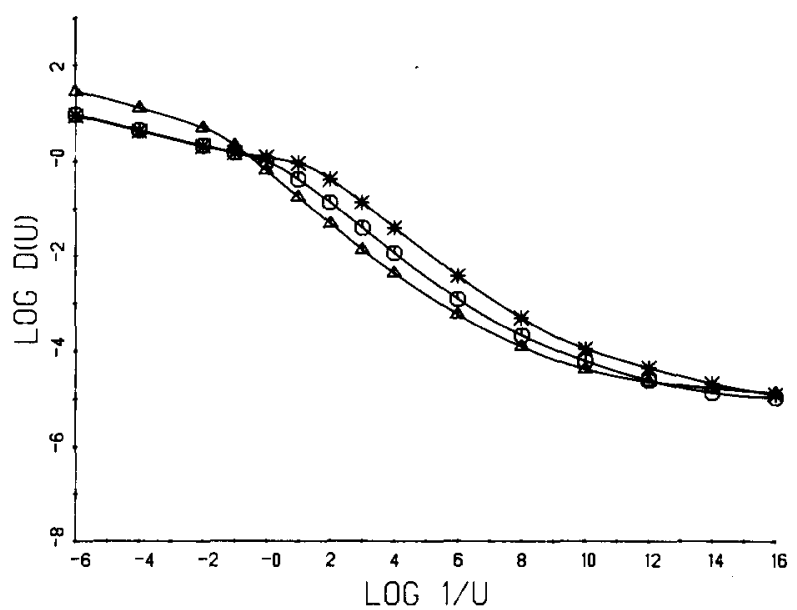

FIG. 11. $D(E, u)$ vs $u$ for $\beta=9, E=0$, dipole-dipole transfer, and: triangles: $\phi=1.0$; circles: $\phi=0.01$; stars: $\phi=0.0001$.

these initial conditions join. An intriguing feature of Fig. 10 is the rather weak dependence of the long-time behavior on the interaction range parameter $s$. Although the shortrange interaction does give significantly slower energy relaxation at long times, and thus a slower approach to the equilibrium diffusion coefficient, the qualitative form of the approach in the range $1 / u=1-10^{8}$ is similar in the two cases (taking into account the different equilibrium values being approached). One should note that the interaction parameters have been chosen so that the two transfer rates are equal at the Förster radius $R=1$ [Eq. (4.2)]; the octupole rate therefore gives faster transport and faster energy relaxation at short times (compare Figs. 6 and 7). Our observations lend support to the generally accepted belief that the dispersion in the site energies is much more important than spatial disorder in this type of system.

The dependence of the spatial transport upon the off-resonance parameter $\phi$ (Fig. 11) is instructive. A larger $\phi$ gives a greater average transfer rate, so that increasing $\phi$ increases both the equilibrium diffusion coefficient and the coefficient of the short-time power law. One sees from Fig. 11, however, that in between these limits a smaller $\phi$ gives faster spatial transport at low temperatures, because it decreases the rate of energy relaxation and hence keeps the excitations in higher-energy sites. Indeed, changing $\phi$ simply shifts the effective time scale in the intermediate time regime. At higher temperatures $(\beta=5)$, the system equilibrates quickly so that this effect is not seen.

Before concluding this section, we make two comments: first, the existence of long-time dispersive transport in energetically disordered systems has recently been experimentally demonstrated. ${ }^{9.27}$ Second, our results agree with the predictions of the Scher-Montroll continuoustime random walk theory ${ }^{28}$ over a wide range of time scales. The latter theory is highly phenomenological: a waiting-time distribution having a power-law tail is postulated, and then used to derive the transport properties. We have thus provided a microscopic justification of that theory for this type of system. 


\section{GENERAL DISCUSSION}

The variety of examples discussed above indicates the wide range of behavior that can be encompassed by our theory. One may argue that the qualitative features of the results are determined primarily by the input parameters $f(E)$, and $\phi(E)$, and not by the details of the dynamics; any energy-space master equation of the form (3.1), which could be written down immediately from physical considerations, would do just as well. In response, we emphasize that we have not simply used a phenomenological master equation; we have obtained the generalized rates $K\left(E, E^{\prime}, u\right)$ from a microscopic calculation. Without the latter, the short-time, non-Markovian dynamics could not have been obtained at all. It is true, however, that in order to complete the theoretical work by predicting the dynamics of an experimental system one must have some knowledge of $f$ and $\phi$. For the simplest example, a two-component solution in which the components are different chemical species, this is no problem; the component concentrations are chosen beforehand and $\phi(E)$ can be determined from the spectra of the individual components via the Förster prescription (a recent review ${ }^{6}$ discusses the application of self-consistent transport theories to experiments on solutions; as described there, in favorable cases no adjustable parameters are required.) The much more interesting systems having a continuous distribution of energies are much harder to deal with. As we said above, $f(E)$ can often be deduced from the absorption spectrum, but the details of $\phi(E)$ are essentially inaccessible. Our results show that the fine details do not appear to be important at long times, so one may hope to test the basic correctness of the theory with the long-time results, and then use the short-time results to determine $\phi(E)$. As we have emphasized, the theory presented here assumes that spatial and energetic disorder are uncorrelated. This necessarily limits its utility for glasses and thin films composed of excitation-carrying molecules, but one can still be reasonably confident about using it for systems in which the excitation conductors are doped into an amorphous structure such as a glass or a polymer matrix.

Our results appear to be broadly consistent with those recently presented by Grünewald et al., ${ }^{14}$ although a precise comparison is not possible since both the underlying theory and the choice of examples are different from ours. These workers study energy relaxation by calculating the mean energy of what we have called an excitation packet, rather than the full distribution; they also find "deceleration" and interpret it in a similar way. Their spatial transport results, presented in the form of a time-dependent mobility, qualitatively resemble ours. In the published work, only the case $\phi=1$ and only exchange interactions are treated (thus, there is no shorttime Haan-Zwanzig asymptote); however, we have been informed ${ }^{29}$ that dipole-dipole interactions give similar results, in conformity with our experience with dipoledipole and octupole-octupole interactions. In any event, we believe that such differences as are seen arise from the models used rather than from the underlying theories, which although not equivalent are similar in spirit. A particularly valuable feature of Ref. 14 is a comparison to exact numerical results: the agreement is quite impressive. Overall, we are pleased to see that not only our qualitative results, but also our physical interpretations, are supported by this quite independent study.

In conclusion, we believe that the theory described here provides a simple yet powerful approach to excitation transport in energetically disordered systems. The calculations, while not trivial, are vastly simpler than computer simulations for systems having long-range transfer rates and complicated site-energy distributions; moreover, simulations are just as dependent upon the unknown parameters $\phi(E)$ and upon assumptions about correlations between spatial and energetic disorder, and so do not avoid the most important limitations of this work.

\section{ACKNOWLEDGMENTS}

We wish to thank Professor H. Bässler for a helpful discussion. This work was supported by NIH Grant 2 RO1 NS08116-16.

\section{APPENDIX A: TWO-BODY APPROXIMATION FOR THE SELF-ENERGY}

When energetic disorder is present, the summation of all two-body self-energy diagrams requires a procedure different from, but scarcely more complicated than, that described by GAF. We refer the reader to Ref. 1 for a discussion of the notation. The basic unit from which a diagram is constructed (Fig. 12) is a factor $w_{21} G_{1}^{s}$, denoted by a dot in circle 1 and a solid arrow from circle 1 to circle 2 [we are using a shorthand notation $w_{12}=w\left(\mathbf{r}_{1}\right.$ $\left.\left.-\mathbf{r}_{2}, E_{1}, E_{2}\right)\right]$. One also has factors of $\left(-w_{21} G_{1}^{s}\right)$, denoted by a dot in circle 1, a solid arrow to 2 , and a dashed arrow back to 1 , which arise from the diagonal part of the master equation. The GAF rules specify that all headto-tail combinations of these units are allowed, yielding diagrams such as those shown in Fig. 12; this leads to rather involved formulas when $w_{12} \neq w_{21}$. However, one can readily see that the two-body diagrams may be calculated by deleting the dashed arrows and instead
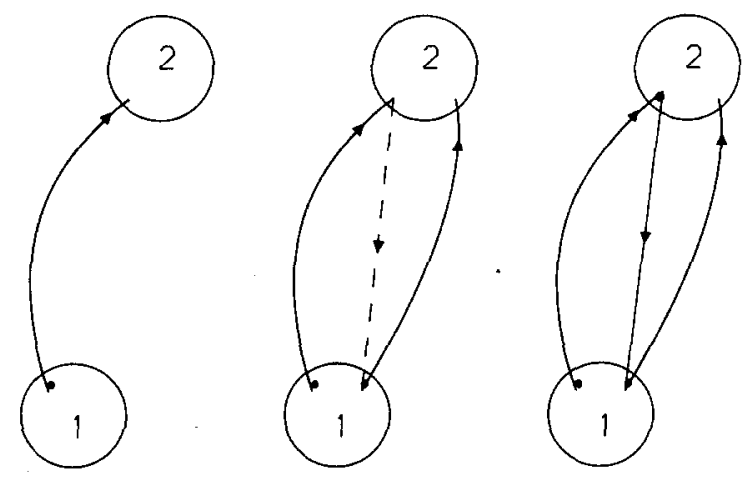

FIG. 12. Examples of two-body self-energy diagrams are shown. These are evaluated as, from left to right, $w_{21} G_{1}^{s},-\left(w_{21} G_{1}^{s}\right)^{2}$, and $\left(w_{21} G_{1}^{s}\right)^{2} w_{12} G_{2}^{s}$. The notation is discussed in the text. 
admitting both head-to-tail and head-to-head combinations of solid arrows, at the same time associating a minus sign with every diagram having an even number of arrows overall. The combinatorial problem becomes simply the number of ways of laying down arrows (with a dot at the tail of each arrow) across two circles. In an $n$th order diagram there are two choices (two directions) for $(n-1)$ arrows, since the orientation of one arrow is fixed by the label on the diagram, i.e., $S_{21} G_{1}^{s}$ or $S_{12} G_{2}^{s}$. The sum of all $n$th order diagrams is therefore evaluated as

$$
\rho S_{12}^{(n)}=w_{12}(-1)^{n-1}\left(w_{12} G_{2}^{s}+w_{21} G_{1}^{s}\right)^{n-1}
$$

so that the sum of all two-body diagrams is

$$
\rho S_{12}=\frac{w_{12}}{1+w_{12} G_{2}^{s}+w_{21} G_{1}^{s}}
$$

whose Fourier transform gives $\Sigma\left(k, E, E^{\prime}\right)$.

\section{APPENDIX B: DIAGONAL APPROXIMATION FOR $G^{*}(E, t)$}

If all off-diagonal elements of the master equation are neglected, the configuration-averaged diagonal Green function becomes

$$
\begin{aligned}
& G^{s}(E, t) \\
& \simeq V^{1-N} \prod_{j=0}^{N-1} \int d E_{j} f\left(E_{j}\right) \tilde{\delta}\left(E-E_{0}\right) \int d r_{j} \delta\left(\mathbf{r}-\mathbf{r}_{0}\right) \\
& \quad \times \exp \left[-t \sum_{1=1}^{N} w\left(\mathbf{r}_{1}-\mathbf{r}_{0}, E_{1}-E_{0}\right)\right]
\end{aligned}
$$

In the thermodynamic and continuum limits, a wellknown procedure ${ }^{16}$ leads to

$$
\begin{aligned}
G^{s}(E, t) & \\
= & {\left[1-\frac{1}{V} \int d \mathbf{r} \int d E^{\prime} f\left(E^{\prime}\right)\left(1-e^{-t w\left(r, E^{\prime}, E\right)}\right)\right]^{\rho V} } \\
= & \exp \left[-\rho \int d E^{\prime} f\left(E^{\prime}\right) \int d \mathbf{r}\left(1-e^{-t w\left(r, E^{\prime}, E\right)}\right)\right] .
\end{aligned}
$$

This approximation provides an extremely useful initial estimate for the numerical solution of Eq. (2.13). It is best at short times, but even at long times it works well enough, provided the temperature is not too low. For transfer rates arising from multipolar interactions, Eq. (4.2), one finds

$G^{s}(E, t)$

$$
\simeq \exp \left\{\frac{-\pi^{d / 2}}{\Gamma[1+(d / 2)]} R^{d} \Gamma\left(1-\frac{d}{s}\right) b(E)(t / \tau)^{d / s}\right\}
$$

in $d$ spatial dimensions, where $\Gamma$ is the usual $\Gamma$ function and

$$
b(E) \equiv \rho \int d E^{\prime} f\left(E^{\prime}\right)\left[e^{\beta\left(E-E^{\prime}\right) / 2} \phi\left(E-E^{\prime}\right)\right]^{d / s}
$$

In the three-dimensional dipole-dipole case $(d=3, s$ $=6$ ) the Laplace transform of $G^{s}(E, t)$, which is used in the calculations, can be performed:

$$
G^{s}(E, u)=\frac{1}{u}\left\{1-\sqrt{\pi} y(u, E) e^{[y(u, E)]^{2}} \operatorname{Erfc}[y(u, E)]\right\},
$$

where Erfc is the complementary error function and

$$
y(u, E) \equiv \frac{2}{3} \pi^{3 / 2} R^{3}(u \tau)^{-1 / 2} b(E) .
$$

For $u=0$, Eq. (B5) reduces to

$$
G^{s}(E, u=0)=\frac{9 \tau}{8\left[\pi R^{3} b(E)\right]^{2}} .
$$

In the octupole case, the transform must be performed numerically.

${ }^{1}$ C. R. Gochanour, H. C. Andersen, and M. D. Fayer, J. Chem. Phys. 70, 4259 (1979).

${ }^{2}$ R. F. Loring, H. C. Andersen, and M. D. Fayer, J. Chem. Phys. 76, 2015 (1982); R. F. Loring and M. D. Fayer, Chem. Phys. 70, 139 (1982).

${ }^{3}$ R. F. Loring, H. C. Andersen, and M. D. Fayer, Phys. Rev. Lett. 50, 1324 (1983); J. Chem. Phys. 80, 5731 (1984).

${ }^{4}$ M. Lax and T. Odagaki, in Macroscopic Properties of Disordered Media, edited by R. Bumridge, S. Childress, and G. Papanicolaou (Springer, Berlin, 1983); I. Webman, Phys. Rev. Lett. 47, 1496 (1981); G. Korzeniewski, R. Friesner, and R. Silbey, J. Stat. Phys. 31, 451 (1983); M. Sahimi, B. D. Hughes, L. E. Scriven, and H. T. Davis, J. Chem. Phys. 78, 6849 (1983).

${ }^{3}$ B. Movaghar and W. Schirmacher, J. Phys. C 14, 859 (1981); B. Movaghar, M. Grünewald, B. Pohlmann, D. Würtz, and W. Schirmacher, J. Stat. Phys. 30, 315 (1983).

${ }^{6}$ M. Ediger and M. D. Fayer, J. Phys. Chem. (in press).

' S. T. Gentry and R. Kopelman, J. Chem. Phys. 78, 373 (1983); 81, 3014, 3022 (1984).

${ }^{8} \mathrm{H}$. Dubost and R. Charneau, Chem. Phys. 12, 407 (1976).

${ }^{9}$ R. Jankowiak and H. Bässler, Chem. Phys. 74, 57 (1983); R. Richert, B. Ries, and H. Bässler, Philos. Mag. B 49, L25 (1984).

${ }^{10}$ K. D. Rockwitz and H. Bässler, Chem. Phys. 70, 307 (1982).

${ }^{11}$ J. Morgan and M. A. El-Sayed, J. Phys. Chem. 87, 2179 (1983).

${ }^{12}$ S. W. Haan and R. Zwanzig, J. Chem. Phys. 68, 1879 (1978).

${ }^{13}$ R. Parson, Chem. Phys. Lett. 99, 213 (1983); R. Parson and R. Kopelman, J. Phys. Chem. 88, 2931 (1984).

${ }_{14}$ M. Grünewald, B. Pohlmann, B. Movaghar, and D. Würtz, Philos. Mag. B 49, 341 (1984).

${ }^{15}$ E. N. Economou, Green's Functions in Quantum Physics (Springer, Berlin, 1983).

${ }^{16}$ M. Inokuti and F. Hirayama, J. Chem. Phys. 43, 1973 (1965).

${ }^{17}$ K. Godzik and J. Jortner, J. Chem. Phys. 72, 4471 (1980); A. Blumen, J. Klafter, and R. Silbey, ibid. 72, 5320 (1980).

${ }^{18}$ R. Zwanzig, in Lectures in Theoretical Physics (Boulder) (Interscience, New York, 1961), Vol. 3, p. 106.

${ }_{19}^{19}$ J. Klafter and R. Silbey, Philos. Mag. B 47, 337 (1983).

${ }^{20} \mathrm{~K}$. Brown and S. Conte, in Proceedings of the 22nd National Conference of the ACM (Thompson, Washington, D.C., 1967).

${ }_{21}$ T. Odagaki and F. Yonezawa, Solid State Commun. 27, 1203 (1978). ${ }^{22}$ V. Agranovich and M. D. Galanin, Electronic Excitation Energy Transfer in Molecular Crystals (North-Holland, New York, 1982).

${ }^{23}$ H. Stehfest, Commun. ACM 13, 47, 624 (1970).

${ }^{24} \mathrm{M}$. Abramowitz and I. E. Stegun, Handbook of Mathematical Functions (Dover, New York, 1965).

${ }^{25}$ F. Forsythe and C. B. Moler, Computer Solution of Linear Algebraic Systems (Prentice-Hall, Englewood Cliffs, 1967).

${ }^{26}$ N. G. Van Kampen, Stochastic Processes in Physics and Chemistry (North-Holland, Amsterdam, 1981).

${ }^{27}$ L. Harmon and R. Kopelman, J. Lumin. (in press); I. Newhouse and R. Kopelman (in press).

${ }^{28} \mathrm{H}$. Scher and E. Montroll, Phys. Rev. B 12, 2465 (1975).

${ }^{29}$ H. Bässler (personal communication June 1984). 